\title{
NEW PROPERTY OF QUATERNIONIC FUETER FUNCTIONS
}

\author{
Wiestaw Królikowski \\ Institute of Mathematics, Czestochowa University of Technology, Czestochowa, Poland \\ wieslaw_krolikowski@wp.pl
}

\begin{abstract}
We present the result of the theory of quaternionic Fueter regular functions. It is shown that the module of such function $\|F(q)\|, q:=w+i x+j y+k z$, cannot be an analytic function in $r ; r^{2}:=\|q\|^{2}:=w^{2}+x^{2}+y^{2}+z^{2}$.
\end{abstract}

Keywords: quaternionic analysis, regular function in the sense of Fueter.

\section{Introduction}

The presented paper concentrates on a special class of mappings from the quaternionic analysis. One of the main problems of the quaternionic analysis is finding an analog of a holomorphic function from the complex analysis in the quaternionic case. It is well known that there are many equivalent conditions for holomorphy in complex analysis. It turned out (which was shown by several authors) that the transfer of such conditions to the quaternions gives in each case different classes of functions (see e.g. [1]). The best promising attempt was the definition of "quaternionic holomorphy", proposed in 1935 by R. Fueter [2], which generalized the Cauchy-Riemann equations. Henceforth these mappings appeared in the literature as "regular mappings in the sense of Fueter". They have many properties "analogous" to those of holomorphic mappings although the proofs are very difficult from the technical point of view because, among other things, of the non-commutativity of quaternions. In many cases the analogy does not exist, for example, there are no "simple" functions which are regular in the sense of Fueter. In 1979 Sudbery [3] collected, classified and proved the most of fundamental properties of regular mappings. In any event, up to now, there does not exist a "quaternionic analysis" in the same sense as the complex analysis, nevertheless in the 1970s Imaeda [4] presented an exceptionally beautiful, simple and convincing application of quaternions to electromagnetism.

Denote by $\mathbf{H}$ the skew field of quaternions. An arbitrary element of $\mathbf{H}$ can be written as $q:=w+i x+j y+k z, w, x, y, z \in \mathbf{R}$ and the quaternionic units satisfy: $i^{2}=j^{2}=k^{2}=i j k=-1$. The conjugation of $\mathbf{H}$ given by

$$
\bar{q}:=w-i x-j y-k z
$$


and the norm $\|q\|:=(q \cdot \bar{q})^{\frac{1}{2}}=(\bar{q} \cdot q)^{\frac{1}{2}}$ can be used to express the inverse element: for $q \in \mathbf{H}, q \neq 0$

$$
q^{-1}:=\frac{\bar{q}}{\|q\|^{2}}
$$

Recall some fundamental facts.

Definition 1 ([1]). A function $f: \mathbf{H} \rightarrow \mathbf{H}$ is called (left) differentiable at $q$ if the limit

$$
\frac{d f}{d q}(q):=\lim _{h \rightarrow 0} h^{-1}[f(q+h)-f(q)]
$$

exists.

It is the most natural definition at first sight but it leads to a very restricted class of functions. Namely, we have

Theorem 1 ([1]). If $\frac{d f}{d q}(q)$ exists, then $f(q)=a+q b, a, b \in \mathbf{H}$.

Quaternions do not commute, hence the reasonable generalization of the term $a_{n} z^{n}$ from the complex case is the form

$$
a_{0} q a_{1} q \ldots a_{n} q a_{n+1}, \quad a_{i} \in \mathbf{H}, i=1,2, \ldots, n+1 .
$$

But the definition of holomorphicity using sums of such terms leads to a quite general class of functions, namely to the real-analytic mappings from $\mathbf{R}^{4}$ to $\mathbf{R}^{4}$.

In 1935 R. Fueter [2] proposed a definition of "regularity" for quaternionic functions via an analogue of the Cauchy- Riemann equations.

A function $F: \mathbf{H} \rightarrow \mathbf{H}$ of the quaternionic variable $q$ can be written as

$$
F:=F_{o}+i F_{1}+j F_{2}+k F_{3} .
$$

$F_{o}$ is called the real part of $F$ and $i F_{1}+j F_{2}+k F_{3}$ - the imaginary part of $F$.

In [2] Fueter introduced the following operators:

$$
\begin{aligned}
\bar{\partial}_{\text {left }} & :=\frac{1}{4}\left(\frac{\partial}{\partial w}+i \frac{\partial}{\partial x}+j \frac{\partial}{\partial y}+k \frac{\partial}{\partial z}\right), \\
\bar{\partial}_{\text {right }} & :=\frac{1}{4}\left(\frac{\partial}{\partial w}+\frac{\partial}{\partial x} i+\frac{\partial}{\partial y} j+\frac{\partial}{\partial z} k\right),
\end{aligned}
$$

analogous to $\frac{\partial}{\partial \bar{z}}=\frac{1}{2}\left(\frac{\partial}{\partial x}+i \frac{\partial}{\partial y}\right)$ in the complex analysis, to generalize the Cauchy-Riemann equations.

Definition 2 ([2]). A quaternionic function $F$ is said to be left regular in the sense of Fueter (respectively: right regular) if it is differentiable in the real variable sense 
and satisfies the condition:

$$
\bar{\partial}_{\text {left }} \cdot F=0 \quad\left(\text { resp. } \bar{\partial}_{\text {right }} \cdot F=0\right) \text {. }
$$

Note that the condition (1) is equivalent to the following system of equations:

$$
\begin{aligned}
& \partial_{w} F_{o}-\partial_{x} F_{1}-\partial_{y} F_{2}-\partial_{z} F_{3}=0, \\
& \partial_{w} F_{1}+\partial_{x} F_{o}+\partial_{y} F_{3}-\partial_{z} F_{2}=0, \\
& \partial_{w} F_{2}-\partial_{x} F_{3}+\partial_{y} F_{o}+\partial_{z} F_{1}=0, \\
& \partial_{w} F_{3}+\partial_{x} F_{2}-\partial_{y} F_{1}+\partial_{z} F_{o}=0 .
\end{aligned}
$$

There are many examples of left (right) regular functions in the sense of Fueter. Many papers have been devoted to study the properties of those functions (see e.g. $[1,2,3,5]$. One has found quaternionic generalizations of the Cauchy theorem, the Cauchy integral formula, Taylor sequence in terms of special polynomials etc.

The main aim of the paper is to prove the following

Theorem 2 (Main Theorem). The Fueter equation (1) does not admit any nontrivial solution whose module would be of the form:

$$
\|F\|:=g(r), \quad r^{2}:=w^{2}+x^{2}+y^{2}+z^{2},
$$

where $g(r)$ is an analytic function in $r$ different from a constant.

To do that we need one important property of the regular mappings in the sense of Fueter.

\section{Important property of the Fueter's regular functions}

This is a result of Sudbery [3] and it can be described as follows.

Let $\nu$ be an unordered set of $n$ integers $\left\{i_{1}, \ldots, i_{n}\right\}$ with $1 \leqslant i_{r} \leqslant 3 ; \nu$ is determined by three integers $n_{1}, n_{2}$ and $n_{3}$ with $n_{1}+n_{2}+n_{3}=n$, where $n_{1}$ is the number of 1 's in $\nu, n_{2}$ - the number of 2's and $n_{3}$ - the number of 3's in $\nu$.

There are $\frac{1}{2}(n+1)(n+2)$ such sets $\nu$ and we denote the set of all of them by $\sigma_{n}$.

Let $e_{i_{r}}$ and $x_{i_{r}}$ denote $i, j, k$ and $x, y, z$ according as $i_{r}$ is 1,2 and 3 , respectively.

Then one defines the following polynomials:

$$
P_{\nu}(q):=\frac{1}{n !} \sum\left(w e_{i_{1}}-x_{i_{1}}\right) \cdot \ldots \cdot\left(w e_{i_{n}}-x_{i_{n}}\right),
$$

where the sum is taken over all $n ! \cdot n_{1} ! \cdot n_{2} ! \cdot n_{3} !$ different orderings of $n_{1} 1$ 's, $n_{2} 2$ 's and $n_{3} 3$ 's; for $n=0$, so $\nu=\emptyset$, we put $P_{\emptyset}(q):=1$.

For example we present the explicit forms of the polynomials $P_{\nu}$ of the first and 
second degrees. Thus we have

$$
\begin{aligned}
& P_{1}=w i-x, \\
& P_{2}=w j-y, \\
& P_{3}=w k-z, \\
& P_{11}=\frac{1}{2}\left(x^{2}-w^{2}\right)-x w i, \\
& P_{12}=x y-w y i-w x j, \\
& P_{13}=x z-w z i-w x k \text {, } \\
& P_{22}=\frac{1}{2}\left(y^{2}-w^{2}\right)-y w j, \\
& P_{23}=y z-w z j-w y k, \\
& P_{33}=\frac{1}{2}\left(z^{2}-w^{2}\right)-z w k .
\end{aligned}
$$

In [3] Sudbery proved the following

Proposition I.1. Suppose $F$ is a left regular function in a neighbourhood of the origin $0 \in \mathbf{H}$. Then there is a ball $B:=B(0, r)$ with center 0 and radius $r$ in which $F(q)$ is represented by a uniformly convergent series

$$
F(q)=\sum_{n=0}^{\infty} \sum_{\nu \in \sigma_{n}} P_{\nu}(q) a_{\nu}, \quad a_{\nu} \in \mathbf{H}
$$

Let $F$ satisfy the assumptions of the above Proposition I.1. Then,

$$
F(q)=a_{o}+\sum_{i=1}^{3} P_{i} \cdot a_{i}+\sum_{i \leqslant j} P_{i j} \cdot a_{i j}+\sum_{i \leqslant j \leqslant k} P_{i j k} \cdot a_{i j k}+\ldots
$$

and

$$
\overline{F(q)}=\overline{a_{o}}+\sum_{i=1}^{3} \overline{a_{i}} \cdot \overline{P_{i}}+\sum_{i \leqslant j} \overline{a_{i j}} \cdot \overline{P_{i j}}+\sum_{i \leqslant j \leqslant k} \overline{a_{i j k}} \cdot \overline{P_{i j k}}+\ldots
$$

We used the following relation (which is easy to verify):

$$
\overline{q_{1} \cdot q_{2}}=\overline{q_{2}} \cdot \overline{q_{1}}, \quad q_{1}, q_{2} \in \mathbf{H} .
$$

Multiplying the expressions (I.1) and (I.2) we get

$$
\begin{aligned}
\|F(q)\|^{2} & =\left\|a_{o}\right\|^{2}+\sum_{i=1}^{3}\left(P_{i} \cdot a_{i} \cdot \overline{a_{o}}+a_{o} \cdot \overline{a_{i}} \cdot \overline{P_{i}}\right) \\
& +\sum_{i \leqslant j}\left(P_{i j} \cdot a_{i j} \cdot \overline{a_{o}}+a_{o} \cdot \overline{a_{i j}} \cdot \overline{P_{i j}}\right)+\sum_{i, j} P_{i} \cdot a_{i} \cdot \overline{a_{j}} \cdot \overline{P_{j}} \\
& +\sum_{i \leqslant j \leqslant k}\left(P_{i j k} \cdot a_{i j k} \cdot \overline{a_{o}}+a_{o} \cdot \overline{a_{i j k}} \cdot \overline{P_{i j k}}\right) \\
& +\sum_{m=1}^{3} \sum_{i \leqslant j}\left(P_{m} \cdot a_{m} \cdot \overline{a_{i j}} \cdot \overline{P_{i j}}+P_{i j} \cdot a_{i j} \cdot \overline{a_{m}} \cdot \overline{P_{m}}\right)+\ldots
\end{aligned}
$$




\section{Proof of Main Theorem}

Assume that $F$ is a solution of the Fueter equation (1) and

$$
\|F(q)\|=g(r), \quad r^{2}:=w^{2}+x^{2}+y^{2}+z^{2},
$$

where $g(r)$ is an analytic function different from a constant in some neighbourhood of $r=0$, i.e. we have

$$
g^{2}(r):=b_{o}+b_{1} r+b_{2} r^{2}+\ldots+b_{n} r^{n}+\ldots
$$

Comparing (I.3) and (I.4) we get

$$
\begin{gathered}
b_{o}=\left\|a_{o}\right\|^{2}, \\
b_{1} \cdot r=\sum_{i=1}^{3}\left(P_{i} \cdot a_{i} \cdot \overline{a_{o}}+a_{o} \cdot \overline{a_{i}} \cdot \overline{P_{i}}\right), \\
b_{2} \cdot r^{2}=\sum_{i \leqslant j}\left(P_{i j} \cdot a_{i j} \cdot \overline{a_{o}}+a_{o} \cdot \overline{a_{i j}} \cdot \overline{P_{i j}}\right)+\sum_{i, j} P_{i} \cdot a_{i} \cdot \overline{a_{j}} \cdot \overline{P_{j}}, \\
b_{3} \cdot r^{3}=\sum_{i \leqslant j \leqslant k}\left(P_{i j k} \cdot a_{i j k} \cdot \overline{a_{o}}+a_{o} \cdot \overline{a_{i j k}} \cdot \overline{P_{i j k}}\right) \\
+\sum_{m=1}^{3} \sum_{i \leqslant j}\left(P_{m} \cdot a_{m} \cdot \overline{a_{i j}} \cdot \overline{P_{i j}}+P_{i j} \cdot a_{i j} \cdot \overline{a_{m}} \overline{P_{m}}\right)
\end{gathered}
$$

etc.

Thus we obtain

$$
b_{O} \geqslant 0
$$

It is easy to verify that the equality (I.6) leads to

$$
a_{1}=a_{2}=a_{3}=0, \quad b_{1}=0 .
$$

Hence, (I.7) can be rewritten as

$$
b_{2} \cdot r^{2}=\sum_{i \leqslant j}\left(P_{i j} \cdot a_{i j} \cdot \overline{a_{o}}+a_{o} \cdot \overline{a_{i j}} \cdot \overline{P_{i j}}\right) .
$$

Consider the equality (I.9). Set

$$
d_{i j}:=a_{i j} \cdot \overline{a_{o}}:=d_{i j}^{o}+\mathbf{i} d_{i j}^{1}+\mathbf{j} d_{i j}^{2}+\mathbf{k} d_{i j}^{3}
$$


(i, j, k denote the quaternionic units) and rewrite (I.9) in the form:

$$
b_{2}\left(w^{2}+x^{2}+y^{2}+z^{2}\right)=2 \cdot \sum_{i \leqslant j} R e\left(P_{i j} \cdot d_{i j}\right) .
$$

Then we get

$$
\begin{aligned}
b_{2}\left(w^{2}+x^{2}+y^{2}+z^{2}\right) & =2 \operatorname{Re}\left\{\left[\frac{1}{2}\left(x^{2}-w^{2}\right)-x w \mathbf{i}\right] d_{11}\right\} \\
& +2 \operatorname{Re}\left\{\left[\frac{1}{2}\left(y^{2}-w^{2}\right)-y w \mathbf{j}\right] d_{22}\right\} \\
& +2 \operatorname{Re}\left\{\left[\frac{1}{2}\left(z^{2}-w^{2}\right)-z w \mathbf{k}\right] d_{33}\right\}+\ldots \\
& =\left(x^{2}-w^{2}\right) d_{11}^{o}+\left(y^{2}-w^{2}\right) d_{22}^{o}+\left(z^{2}-w^{2}\right) d_{33}^{o} .
\end{aligned}
$$

Comparing the terms in $x^{2}, y^{2}$ and $z^{2}$ we obtain:

$$
b_{2}=d_{11}^{o}=d_{22}^{o}=d_{33}^{o}
$$

but then

$$
b_{2} w^{2}=-3 w^{2} b_{2}
$$

Thus

$$
b_{2}=0
$$

and, as a result of (I.9),

$$
a_{i j}=0
$$

The equality (I.8) takes the form

$$
b_{3} \cdot r^{3}=\sum_{i \leqslant j \leqslant k}\left(P_{i j k} \cdot a_{i j k} \cdot \overline{a_{o}}+a_{o} \cdot \overline{a_{i j k}} \cdot \overline{P_{i j k}}\right) .
$$

This implies that

$$
b_{3}=0, \quad a_{i j k}=0
$$

Now we will prove that $b_{4}=0$.

We have

$$
b_{4} \cdot r^{4}=\sum\left(P_{i j k l} \cdot a_{i j k l} \cdot \overline{a_{o}}+a_{o} \cdot \overline{a_{i j k l}} \cdot \overline{P_{i j k l}}\right) .
$$


There are $\frac{1}{2}(4+1)(4+2)=15$ polynomials $P_{i j k l}$. We will write all of them:
1. $P_{1111}=\frac{1}{24}(w i-x)(w i-x)(w i-x)(w i-x)$,
2. $P_{2222}=\frac{1}{24}(w j-y)(w j-y)(w j-y)(w j-y)$,
3. $P_{3333}=\frac{1}{24}(w k-z)(w k-z)(w k-z)(w k-z)$,
4. $P_{1112}=\frac{1}{24}(w i-x)(w i-x)(w i-x)(w j-y)$,
5. $P_{1113}=\frac{1}{24}(w i-x)(w i-x)(w i-x)(w k-z)$,
6. $P_{2223}=\frac{1}{24}(w j-y)(w j-y)(w j-y)(w k-z)$,
7. $P_{1222}=\frac{1}{24}(w i-x)(w j-y)(w j-y)(w j-y)$,
8. $P_{1333}=\frac{1}{24}(w i-x)(w k-z)(w k-z)(w k-z)$,
9. $P_{2333}=\frac{1}{24}(w j-y)(w k-z)(w k-z)(w k-z)$,
10. $P_{1122}=\frac{1}{24}(w i-x)(w i-x)(w j-y)(w j-y)$,
11. $P_{1133}=\frac{1}{24}(w i-x)(w i-x)(w k-z)(w k-z)$,
12. $P_{2233}=\frac{1}{24}(w j-y)(w j-y)(w k-z)(w k-z)$,
13. $P_{1123}=\frac{1}{24}(w i-x)(w i-x)(w j-y)(w k-z)$,
14. $P_{1223}=\frac{1}{24}(w i-x)(w j-y)(w j-y)(w k-z)$,
15. $P_{1233}=\frac{1}{24}(w i-x)(w j-y)(w k-z)(w k-z)$.

The equality (I.10) can be rewritten in the form:

$$
b_{4}\left(w^{2}+x^{2}+y^{2}+z^{2}\right)^{2}=2 \sum R e\left(P_{i j k l} \cdot d_{i j k l}\right),
$$

where

$$
d_{i j k l}:=a_{i j k l} \cdot \overline{a_{o}}
$$

Note that

$$
b_{4}\left(w^{2}+x^{2}+y^{2}+z^{2}\right)^{2}=2 \sum R e\left(P_{i j k l} \cdot d_{i j k l}\right)
$$




$$
\begin{aligned}
=\frac{1}{12}\left[w^{4}+x^{4}+\ldots\right] d_{1111}^{o} & +\frac{1}{12}\left[w^{4}+y^{4}+\ldots\right] d_{2222}^{o}+\frac{1}{12}\left[w^{4}+z^{4}+\ldots\right] d_{3333}^{o} \\
& +\frac{1}{12}\left[w^{4}+\ldots\right] d_{1122}^{o}+\frac{1}{12}\left[w^{4}+\ldots\right] d_{1133}^{o}+\frac{1}{12}\left[w^{4}+\ldots\right] d_{2233}^{o} \\
& =\frac{1}{12} w^{4}\left(d_{1111}^{o}+d_{2222}^{o}+d_{3333}^{o}+d_{1122}^{o}+d_{1133}^{o}+d_{2233}^{o}\right) \\
& +\frac{1}{12} x^{4} d_{1111}^{o}+\frac{1}{12} y^{4} d_{2222}^{o}+\frac{1}{12} z^{4} d_{3333}^{o} .
\end{aligned}
$$

where

$$
d_{i j k l}^{o}:=R e d_{i j k l} .
$$

Comparing the terms in $x^{4}, y^{4}, z^{4}$ and $w^{4}$ we get

$$
\begin{aligned}
b_{4} x^{4} & =\frac{1}{12} d_{1111}^{o} x^{4}, \\
b_{4} y^{4} & =\frac{1}{12} d_{2222}^{o} y^{4}, \\
b_{4} z^{4} & =\frac{1}{12} d_{3333}^{o} z^{4}, \\
b_{4} w^{4} & =\frac{1}{12} w^{4}\left(d_{1111}^{o}+d_{2222}^{o}+d_{3333}^{o}+d_{1122}^{o}+d_{1133}^{o}+d_{2233}^{o}\right) .
\end{aligned}
$$

Comparing the terms in $x^{2} y^{2}, x^{2} z^{2}$ and $y^{2} z^{2}$ we have

$$
\begin{aligned}
& 2 b_{4} x^{2} y^{2}=\frac{1}{24} x^{2} y^{2} d_{1122}^{o} \\
& 2 b_{4} x^{2} z^{2}=\frac{1}{24} x^{2} z^{2} d_{1133}^{o}, \\
& 2 b_{4} y^{2} z^{2}=\frac{1}{24} y^{2} z^{2} d_{2233}^{o} .
\end{aligned}
$$

Thus we get

$$
b_{4}=b_{4}+b_{4}+b_{4}+\frac{1}{12}\left(d_{1122}^{o}+d_{1133}^{o}+d_{2233}^{o}\right)
$$

i.e.

$$
-2 b_{4}=\frac{1}{12}\left(d_{1122}^{o}+d_{1133}^{o}+d_{2233}^{o}\right)
$$

and finally

$$
-2 b_{4}=3 b_{4}
$$


but this is impossible. Then

$$
b_{4}=0, \quad a_{i j k l}=0 .
$$

By the analogous considerations we obtain

$$
b_{1}=b_{2}=b_{3}=b_{4}=\ldots=b_{n}=\ldots=0 .
$$

Thus $g(r)$ has to be constant:

$$
g(r)=\sqrt{b_{o}} .
$$

The last condition contradicts to the assumption that $g(r)$ is different from a constant. This ends the proof.

\section{References}

[1] Królikowski W., On Fueter-Hurwitz Regular Mappings, Dissertationes Mathematicae, Warsaw 1996, CCCLIII.

[2] Fueter R., Die Funktionentheorie der Differentialgleichungen $\Delta u=0$ und $\Delta \Delta u=0$ mit vier reellen Variablen, Comment. Math. Helv. 1935, 7, 307-330.

[3] Sudbery A., Quaternionic analysis, Math. Proc. Cambridge Philos. Soc. 1979, 85, 199-225.

[4] Imaeda K., Quaternionic Formulation of Classical Electrodynamics and Theory of Functions of a Biquaternion Variable, Report of Fundamental Physics Laboratory, Department of Electronic Science, Faculty of Science, Okayama University of Science, Feb., 1983.

[5] Królikowski W., Some Problems of Quaternionic Analysis, Czestochowa University of Technology, Czestochowa 2012. 